\title{
Ultrassonografia transcraniana em cães hígidos: padronização da técnica e descrição anatômica
}

[Transcranial ultrasonography in healthy dogs: technique standardization and anatomic description]

\author{
T.C.F. Cintra ${ }^{1}$, C.F. Carvalho ${ }^{2}$, J.C. Canola ${ }^{1}$, A.C. Nepomuceno ${ }^{1}$ \\ ${ }^{1}$ Universidade Estadual Paulista - Jaboticabal, SP \\ ${ }^{2}$ Universidade Cruzeiro do Sul - São Paulo, SP
}

\begin{abstract}
RESUMO
O interesse pelo estudo do sistema nervoso central vem crescendo na Medicina Veterinária. A ultrassonografia transcraniana (USTC) tem a vantagem de não ser invasiva e ter baixo custo quando comparada à tomografia computadorizada (TC) e à imagem em ressonância magnética (RM). O osso temporal tem sido utilizado como janela acústica na realização da USTC em seres humanos. Este trabalho teve como objetivo correlacionar imagens ultrassonográficas transcranianas obtidas através das janelas temporal e occipital com a anatomia encefálica em cães adultos hígidos, e padronizar a técnica de exame. Foram utilizados 37 cães adultos, sem raça definida: 30 animais in vivo, para realização da técnica, e sete cadáveres, para a secção do encéfalo, à similitude dos planos de imagem obtidos ao USTC. Os dados foram analisados utilizando o teste não paramétrico de Wilcoxon. Os resultados obtidos indicaram que a USTC é um método diagnóstico viável para a avaliação do encéfalo de cães com peso corporal até $10 \mathrm{~kg}$, por meio de planos dorsal e dorsais oblíquos, sem a necessidade de anestesiar o paciente.
\end{abstract}

Palavras-chave: cães, encéfalo, ultrassonografia

\begin{abstract}
The study of the nervous system is increasing in Veterinary Medicine. Transcranial ultrasonography (TCUS) has the advantage of being a non-invasive and low cost method compared to computerized tomography $(C T)$ and Magnetic Resonance Imaging (MRI). The temporal bone has been used as an acoustic window in TCUS in humans. This study aimed to correlate transcranial ultrasonographic images obtained through the temporal and occipital window with healthy dog's encephalic anatomy, and to standardize the technique. 37 adult mongrel dogs were used: 30 animals in vivo, in order to perform USTC screening and seven dog corpses for brain section as well as USTC planes. Data analysis was accomplished by the non parametric Wilcoxon test. Results obtained indicate that TCUS (in dorsal and oblique planes) is a viable method for brain evaluation in dogs weighting up to 10kg without anesthesia.
\end{abstract}

Keywords: dogs, brain, ultrasonography

\section{INTRODUÇÃO}

A ultrassonografia foi o primeiro método de diagnóstico por imagem utilizado na investigação do encéfalo, em seres humanos (Leksell, 1956). Na Medicina Veterinária, constitui um método de grande valor diagnóstico, sobretudo pela rapidez e o baixo custo com que pode ser realizada, sem a necessidade de anestesiar o paciente (Schellinger et al., 1985).

A literatura descreve o uso da ultrassonografia para o diagnóstico da hidrocefalia em cães pela fontanela bregmática persistente (Prynn e Kedding, 1968) e na investigação do encéfalo de animais com suspeita de neoplasia, em que a extensão do tecido neoplásico pode ser

Recebido em 31 de julho de 2012

Aceito em 5 de setembro de 2013

E-mail: tha_cintra@yahoo.com.br 
monitorada por meio do defeito craniano resultante de lise óssea ou de investigação cirúrgica prévia (Hudson et al., 1998). Pode ser utilizada ainda para detectar a presença de hemorragia subaracnóidea ou intraparenquimal, quando houver fratura craniana em consequência de trauma (Hudson et al., 1998).

Alguns autores relataram a utilização do osso temporal como janela acústica. Segundo Hudson et al. (1998), Carvalho et al. (2007) e Lorigados (2008), a abordagem transcraniana pode ser realizada com sucesso em alguns cães mesmo com a fontanela bregmática fechada. Isso porque, em alguns animais, a calota craniana é suficientemente fina, de modo a permitir a transmissão do feixe sonoro através do osso temporal (Lorigados, 2008).

Atualmente, os equipamentos modernos e de alta resolução permitem o estudo ultrassonográfico realizado através da tábua óssea íntegra, com detalhamento anatômico de estruturas normais e a localização de lesões, complementando a TC e a RM, a custos inferiores (Berg et al., 2008; Lorigados, 2008).

No entanto, há na literatura uma lacuna referente à padronização da técnica ultrassonográfica transcraniana em cães como método diagnóstico na rotina médico-hospitalar. Assim sendo, objetivou-se, com este trabalho, correlacionar imagens ultrassonográficas obtidas pelas janelas temporal e occipital com a anatomia do encéfalo em cães adultos, identificando nas imagens ecográficas e macroscópicas as estruturas encefálicas, a fim de avaliar a acurácia da técnica e determinar o padrão de normalidade dos planos de varredura ultrassonográfica.

\section{MATERIAL E MÉTODOS}

Todo procedimento desta pesquisa esteve de acordo com o preconizado pelo Comitê de Ética na Utilização de Animais - CEUA -, sob número 003993-09.

Foram submetidos à ultrassonografia transcraniana (USTC) 37 cães adultos, sem raça definida, mesaticefálicos, pesando entre 2 e $10 \mathrm{~kg}$. Destes, foram utilizados, para a varredura ultrassonográfica transcraniana, 30 animais in vivo, que não apresentaram alterações ao exame clínico, provenientes do Centro de Pesquisa em
Sanidade Animal (CPPAR) e da rotina do Hospital Veterinário Governador Laudo Natel, setores da Universidade Estadual Paulista Júlio de Mesquita Filho (campus de Jaboticabal, SP). Para a secção do encéfalo, à similitude dos planos de varredura ultrassonográficos transcranianos, foram utilizados sete cadáveres submetidos à eutanásia por meio da aplicação de Propofol $(10 \mathrm{mg} / \mathrm{kg})$ seguido da aplicação de $10 \mathrm{ml}$ de Cloreto de Potássio a $10 \%$ por via intravenosa, não apresentando sinais clínicos de desordem neurológica, sob indicação clínica do Setor de Clínica Médica ou Cirúrgica do Hospital Veterinário Governador Laudo Natel FCAV/UNESP - campus de Jaboticabal e do Centro de Controle de Zoonoses da cidade de Ribeirão Preto, SP.

A avaliação ultrassonográfica do encéfalo foi realizada com equipamento ultrassonográfico (modelo SSI - 1000V, SonoScape Co., Ltd., China), equipado com transdutor convexo multifrequencial com frequência variando de 4 a $7 \mathrm{MHz}$.

Para realização do exame ultrassonográfico, os animais foram posicionados em decúbito lateral esquerdo, para o acesso pela janela temporal do lado direito da cabeça, e em decúbito lateral direito, para o acesso do lado esquerdo da cabeça. $\mathrm{O}$ acesso pelo forame magno foi realizado com o animal posicionado em decúbito lateral direito, com a cabeça flexionada ventralmente, formando um ângulo de aproximadamente $30^{\circ}$ entre a mandíbula e o pescoço. Foi realizada tricotomia bilateral nas regiões temporais e na região occipital, seguindo-se aplicação de gel condutor acústico entre o transdutor e a pele do animal.

O transdutor foi posicionado, inicialmente, perpendicular ao osso temporal cranialmente à orelha externa, paralelo e dorsalmente ao processo zigomático, para obtenção do plano dorsal (Fig. 1A) e, ato contínuo, rotacionado $45^{\circ}$ em sentido horário, seguindo-se a realização de cortes, de aproximadamente de $10^{\circ}$ em $10^{\circ}$, em planos dorsais oblíquos caudais, obtidos com movimento em leque do transdutor, no sentido ventrodorsal (Fig. 1B). Em seguida, o transdutor foi rotacionado no sentido anti-horário, $45^{\circ} \mathrm{em}$ relação ao plano dorsal, para obtenção de planos dorsais oblíquos craniais realizados à semelhança dos planos anteriores (Fig. 1C). Para avaliação 
pela janela occipital, o transdutor foi posicionado perpendicular ao forame magno para obtenção de plano longitudinal; em seguida, rotacionado em $90^{\circ} \mathrm{de}$ seu eixo central para plano dorsal, e, finalmente, inclinado em $10^{\circ}$ em relação ao plano anterior para obtenção de plano dorsal oblíquo. Após a USTC nos cadáveres, as cabeças foram removidas e congeladas inicialmente a $30^{\circ} \mathrm{C}$ durante $48 \mathrm{~h}$ e posteriormente a $-70^{\circ} \mathrm{C}$ durante uma semana, segundo técnica descrita por Rodriguez et al. (2007). Após o processo de congelamento, foram seccionadas nos mesmos planos de varredura obtidos, com auxílio de serra elétrica fixa em fita. Posteriormente, as imagens ultrassonográficas foram correlacionadas com os cortes anatômicos para identificação das estruturas encefálicas.

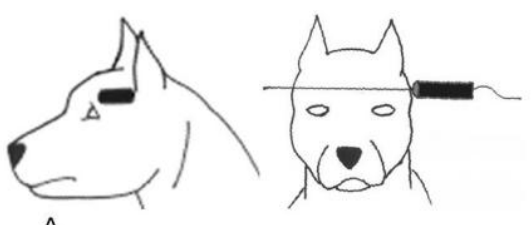

A

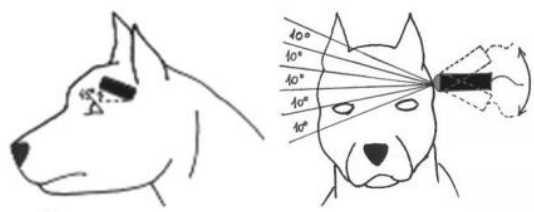

B
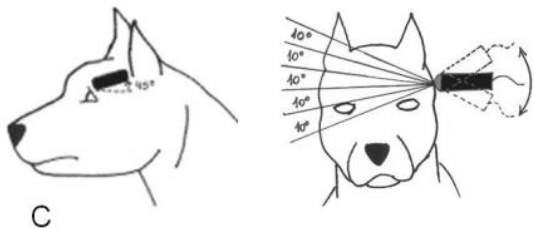

Figura 1. Desenho esquemático ilustrando o posicionamento do transdutor para varredura ultrassonográfica do encéfalo. A) Posicionamento do transdutor perpendicular ao osso temporal para obtenção do plano de secção dorsal mediano. B) Posicionamento do transdutor após rotação de $45^{\circ} \mathrm{em}$ sentido horário e angulação de $30^{\circ} \mathrm{em}$ sentido dorsal para obtenção dos planos dorsais oblíquos caudais. C) Posicionamento do transdutor após rotação de $45^{\circ} \mathrm{em}$ sentido anti-horário e angulação de $30^{\circ} \mathrm{em}$ sentido dorsal para obtenção dos planos dorsais oblíquos craniais.
Posteriormente, o número de estruturas identificadas nos cortes anatômicos foi comparado com o número de estruturas identificadas nos planos ultrassonográficos correspondentes, utilizando o teste não paramétrico de Wilcoxon, com a finalidade de avaliar a acurácia da USTC.

\section{RESULTADOS}

A USTC realizada pela janela temporal em plano dorsal possibilitou a visibilização dos contornos da calota craniana como uma linha hiperecogênica arqueada no topo da imagem, o sulco suprasilviano como uma estrutura linear hiperecogênica se destacando em meio ao tecido cerebral hipoecogênico, os ventrículos laterais anecogênicos, o septo pelúcido como uma fina linha hiperecogênica que separa os dois ventrículos laterais e a fissura longitudinal do cérebro como uma linha hiperecogênica no centro da imagem. Os lobos frontal, temporal e occipital foram identificados de acordo com a topografia da realização do plano de varredura (Fig. 2) (Tab. 1).

Nos planos dorsais oblíquos caudais, foi possível observar os sulcos ectosilviano, suprasilviano, presilviano, esplenial e marginal hiperecogênicos, o tentório ósseo do cerebelo como uma linha hiperecogênica em região caudal da imagem e a fissura longitudinal do cérebro. Os lobos frontal, temporal, parietal e occipital, bem como o cerebelo e o mesencéfalo, foram identificados de acordo com a topografia da realização do plano de varredura (Tab.1) (Fig.3).

Nos planos dorsais oblíquos craniais foram visibilizados ventrículos laterais e septo pelúcido, sulcos presilviano, suprasilviano, ectomarginal, marginal e coronal, tentório ósseo do cerebelo e fissura longitudinal do cérebro. $\mathrm{O}$ mesencéfalo, telencéfalo e diencéfalo foram visibilizados como área de ecogenicidade discretamente reduzida em relação ao restante do parênquima encefálico e identificados de acordo com a topografia do plano de varredura (Tab. 1) (Fig. 4). 


\section{Cintra et al.}
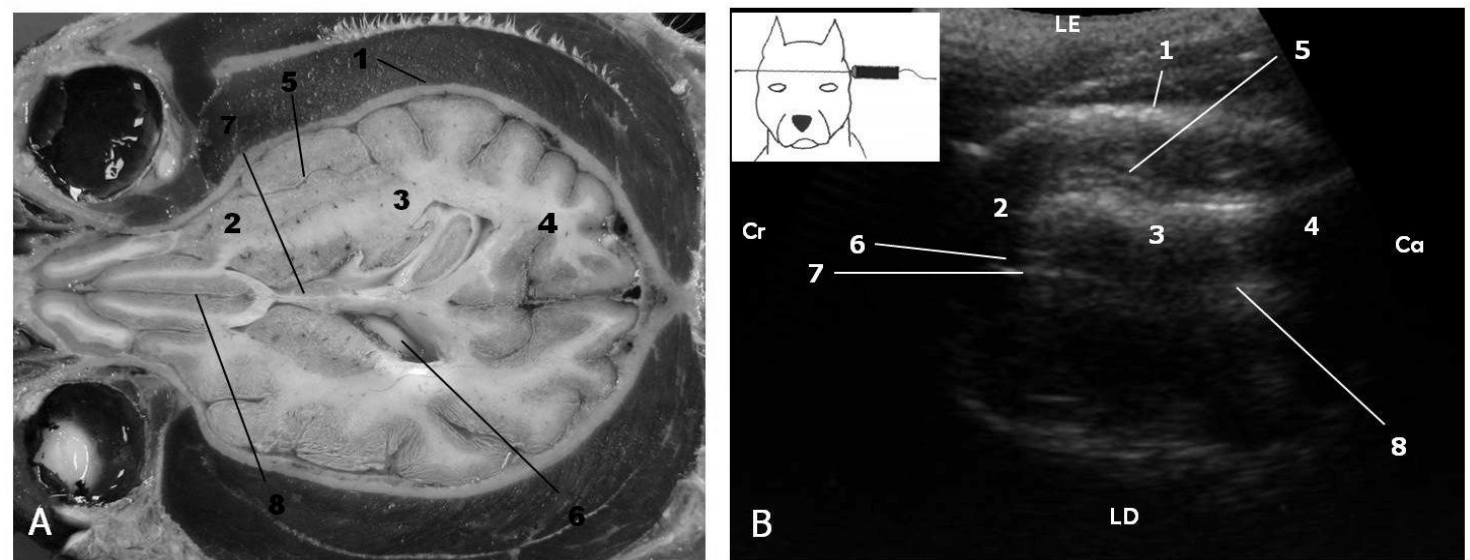

Figura 2. A) Peça anatômica da cabeça de cão em secção correspondente ao plano de imagem dorsal mediano. B) Imagem ultrassonográfica do plano dorsal mediano, com o transdutor posicionado perpendicularmente em relação ao osso temporal. O desenho esquemático no canto superior esquerdo indica a topografia do corte. $1=$ osso temporal; $2=$ lobo frontal; $3=$ lobo temporal; $4=$ lobo occipital; $5=$ sulco suprasilviano; 6 = ventrículo lateral; 7 = septo pelúcido; $8=$ Fissura longitudinal do cérebro; LE = lado esquerdo; $\mathrm{LD}=$ lado direito; $\mathrm{Cr}=$ cranial $; \mathrm{Ca}=$ caudal.

Tabela 1. Denominação dos planos de corte ultrassonográficos, das estruturas encefálicas visibilizadas em cada plano de corte obtido por meio de exame ultrassonográfico realizado pela janela temporal nos 30 animais in vivo e das estruturas visibilizadas nos planos de corte anatômicos correspondentes realizados nos 7 cadáveres. UNESP - Jaboticabal/ 2010

\begin{tabular}{|c|c|c|}
\hline \multirow{2}{*}{$\begin{array}{c}\text { Planos de corte } \\
\text { ultrassonográfico }\end{array}$} & \multicolumn{2}{|c|}{ Estruturas encefálicas visibilizadas } \\
\hline & Corte ultrassonográfico & Corte anatômico \\
\hline Plano dorsal & $\begin{array}{c}\text { Osso temporal, lobos frontal, } \\
\text { temporal e occipital, sulco } \\
\text { suprasilviano, ventrículos laterais, } \\
\text { septo pelúcido, fissura longitudinal } \\
\text { do cérebro }\end{array}$ & $\begin{array}{l}\text { Osso temporal, lobos frontal, temporal e } \\
\text { occipital, sulco suprasilviano, } \\
\text { ventrículos laterais, septo pelúcido, } \\
\text { fissura longitudinal do cérebro, bulbo } \\
\text { olfatório, hipocampo }\end{array}$ \\
\hline $\begin{array}{l}\text { Planos dorsais } \\
\text { oblíquos caudais }\end{array}$ & $\begin{array}{l}\text { Osso temporal, osso parietal, lobos } \\
\text { frontal, temporal, parietal e occipital, } \\
\text { sulco ectosilviano, sulco } \\
\text { suprasilviano, sulco presilviano, sulco } \\
\text { esplenial, sulco marginal, fissura } \\
\text { longitudinal do cérebro, cerebelo, } \\
\text { tentório ósseo do cerebelo, } \\
\text { mesencéfalo, osso etmoide, osso } \\
\text { esfenoide }\end{array}$ & $\begin{array}{l}\text { Osso temporal, osso parietal, lobos } \\
\text { frontal, temporal, parietal e occipital, } \\
\text { sulco ectosilviano, sulco suprasilviano, } \\
\text { sulco presilviano, sulco esplenial, sulco } \\
\text { marginal, sulco ectomarginal, fissura } \\
\text { pseudosilviana, bulbo olfatório, fissura } \\
\text { longitudinal do cérebro, hipocampo, } \\
\text { cerebelo, tentório ósseo do cerebelo, } \\
\text { mesnecéfalo, osso etmoide, osso } \\
\text { esfenoide }\end{array}$ \\
\hline $\begin{array}{l}\text { Planos dorsais } \\
\text { oblíquos craniais }\end{array}$ & $\begin{array}{l}\text { Osso temporal, osso parietal, lobo } \\
\text { frontal, lobo temporal, lobo occipital, } \\
\text { lobo parietal, sulco présilviano, sulco } \\
\text { suprasilviano, sulco ectomarginal, } \\
\text { sulco marginal, sulco coronal, fissura } \\
\text { pseudosilviana, fissura longitudinal } \\
\text { do cérebro, ventrículo lateral, septo } \\
\text { pelúcido, diencéfalo, região de } \\
\text { tálamo, mesencéfalo e cerebelo, } \\
\text { tentório ósseo do cerebelo, osso } \\
\text { basoesfenoidal. }\end{array}$ & $\begin{array}{l}\text { Osso temporal, osso parietal, lobo } \\
\text { frontal, lobo temporal, lobo occipital, } \\
\text { lobo parietal, sulco presilviano, sulco } \\
\text { suprasilviano, sulco ectomarginal, sulco } \\
\text { marginal, sulco coronal, fissura } \\
\text { psudosilviana, bulbo olfatório, fissura } \\
\text { longitudinal do cérebro, ventrículo } \\
\text { lateral, septo pelúcido, hipocampo, } \\
\text { corpo caloso, diencéfalo, região de } \\
\text { tálamo, mesencéfalo e cerebelo, tentório } \\
\text { ósseo do cerebelo, osso basoesfenoidal. }\end{array}$ \\
\hline
\end{tabular}



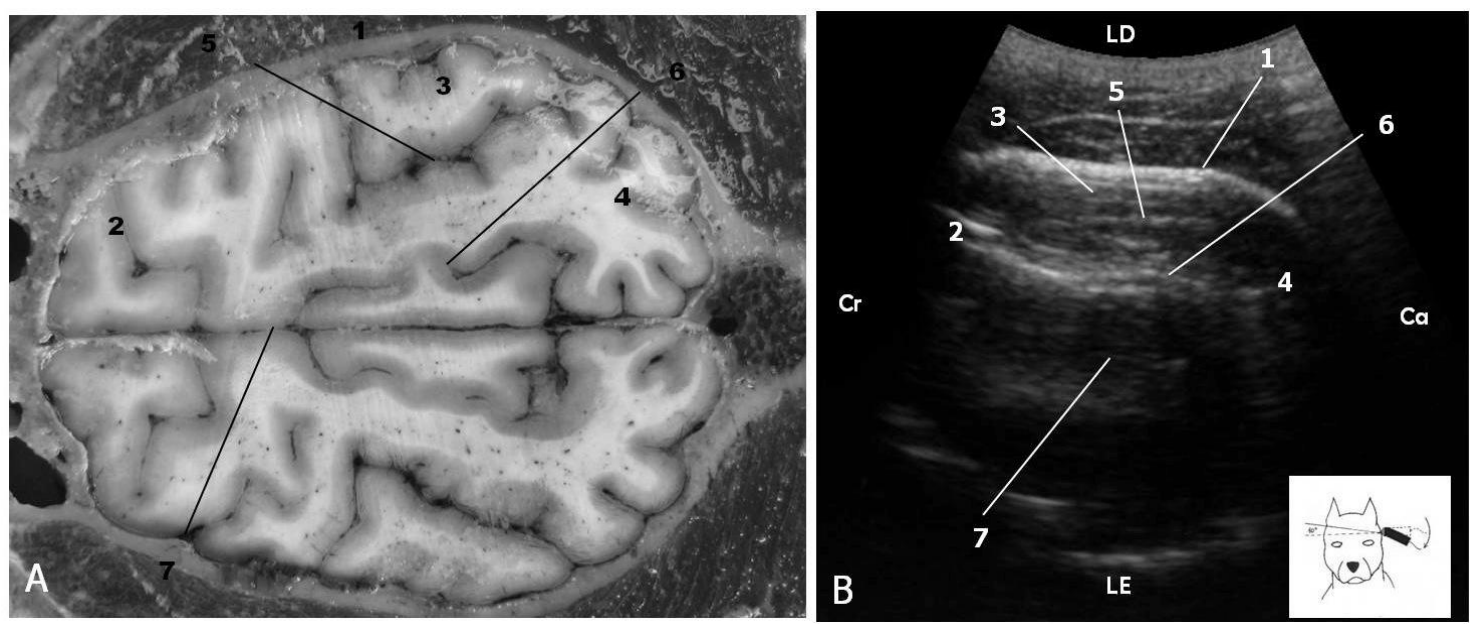

Figura 3. A) Peça anatômica da cabeça de cão em secção correspondente ao plano de dorsal oblíquo caudal demonstrado na imagem ultrassonográfica. B) Plano de imagem dorsal oblíquo caudal através da janela temporal, com o transdutor rotacionado $45^{\circ} \mathrm{em}$ sentido horário e inclinado $10^{\circ} \mathrm{em}$ sentido dorsal. O desenho esquemático no canto inferior direito indica a topografia do corte. $1=$ osso temporal; $2=$ lobo frontal; 3 = lobo temporal; $4=$ lobo occipital; $5=$ sulco suprasilviano; $6=$ sulco esplenial; $7=$ fissura longitudinal do cérebro; $\mathrm{LE}=$ lado esquerdo; $\mathrm{LD}=$ lado direito; $\mathrm{Cr}=$ cranial; $\mathrm{Ca}=$ caudal.
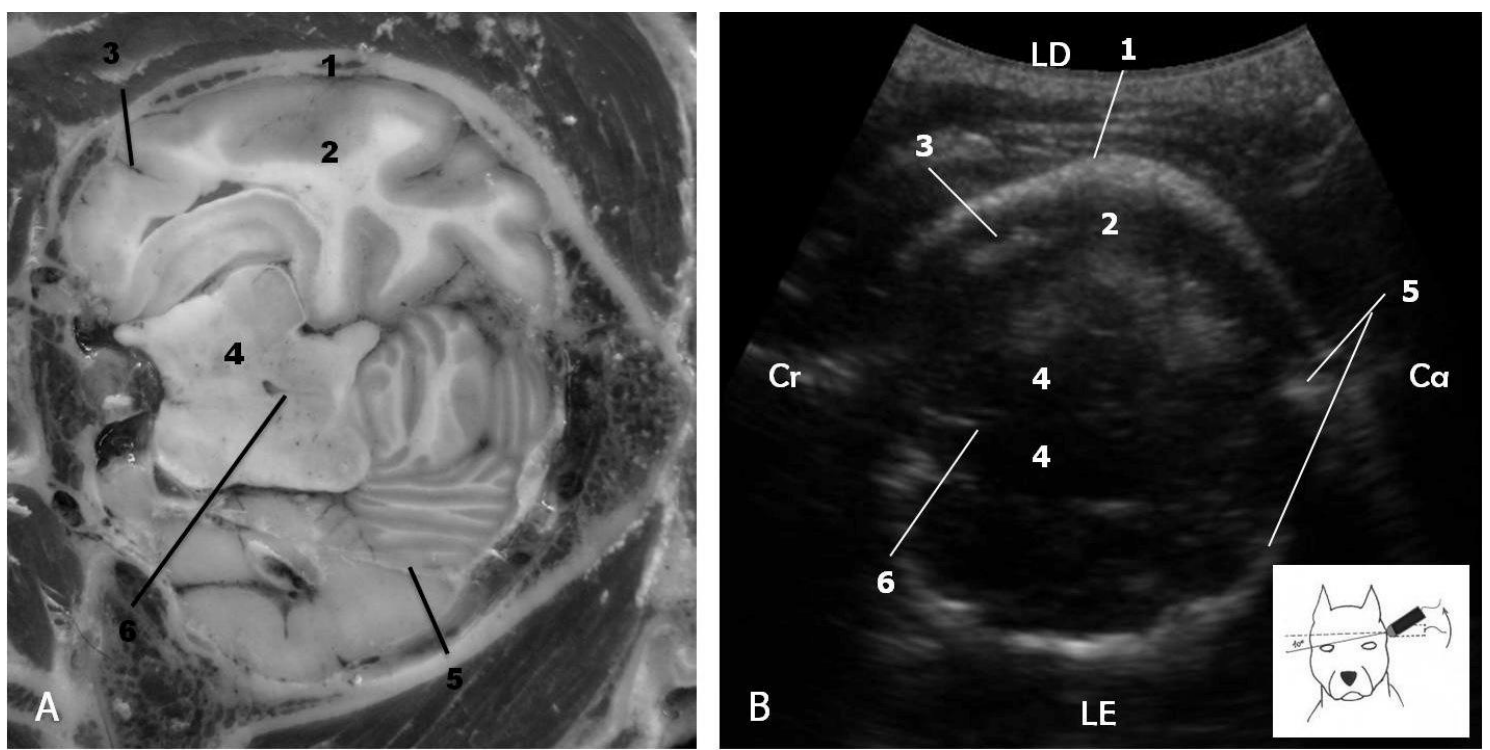

Figura 4. A) Peça anatômica da cabeça de cão em secção correspondente ao plano dorsal oblíquo cranial demonstrado na imagem ultrassonográfica. B) Plano de Imagem dorsal oblíquo cranial através da janela temporal, com o transdutor rotacionado $45^{\circ} \mathrm{em}$ sentido anti-horário e inclinado $10^{\circ} \mathrm{em}$ sentido ventral. $\mathrm{O}$ desenho esquemático no canto inferior direito indica a topografia do corte. $1=$ osso temporal; $2=1$ lobo temporal; 3 = fissura pseudosilviana; 4 = diencéfalo; 5 = tentório ósseo do cerebelo; $6=$ fissura longitudinal do cérebro; $\mathrm{LD}=$ lado direito; $\mathrm{LE}=$ lado esquerdo; $\mathrm{Cr}=$ cranial; $\mathrm{Ca}=$ caudal.

O número de estruturas encefálicas visibilizadas nos planos dorsal e dorsais oblíquos caudais durante a USTC realizada pela janela temporal nos 30 animais in vivo, quando comparado com o número de estruturas observadas nos cortes anatômicos correspondentes, apresentou diferença significativa $(\mathrm{p}<0,05)$. Em contrapartida, não houve diferença significativa ( $p>0,05)$ entre o número de estruturas encefálicas visibilizadas nos planos dorsais oblíquos craniais 
e o número de estruturas observadas nos cortes anatômicos correspondentes.

Por meio da varredura encefálica pela janela occipital, foi possível identificar o cerebelo como uma estrutura hipoecogênica arredondada, os vermes do cerebelo, como linhas paralelas hiperecogênicas, a medula oblonga, como uma estrutura hipoecogênica circundada por área anecogênica correspondente ao espaço subaracnóideo (Fig.5) (Tab.2).
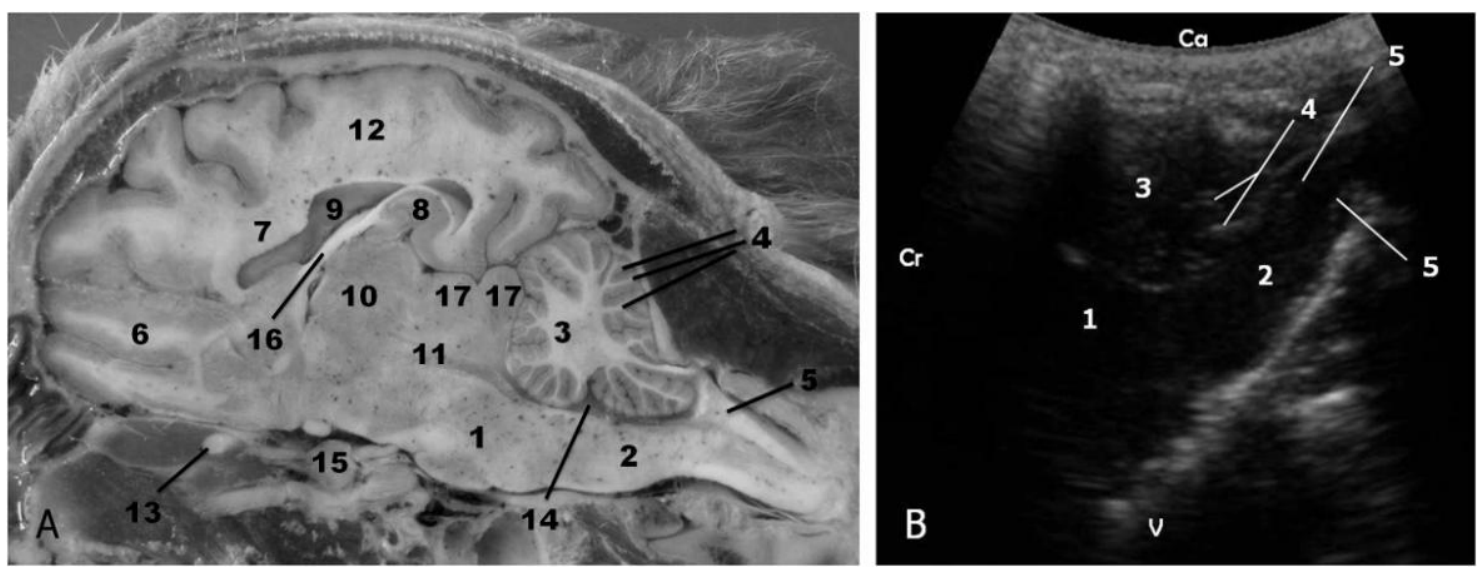

Figura 5. A) Peça anatômica da cabeça de cão em secção correspondente ao plano de imagem longitudinal através da janela occipital. B) Plano de Imagem longitudinal através da janela occipital, com o transdutor posicionado perpendicularmente ao forame magno. $1=$ ponte; $2=$ medula oblonga; $3=$ cerebelo; 4 = vermes do cerebelo; $5=$ Interface entre substância branca e substância cinzenta; $6=$ bulbo olfatório; 7 = corpo caloso; $8=$ hipocampo; 9 = ventrículo lateral; $10=$ diencéfalo; $11=$ mesencéfalo; 12 = hemisfério cerebral; $13=$ nervo óptico; $14=$ IV ventrículo; $15=$ hipófise; 16 = fórnix; 17 = corpos quadrigêmeos; $\mathrm{V}=$ ventral; $\mathrm{Cr}=$ cranial; $\mathrm{Ca}=$ caudal.

Tabela 2. Denominação dos planos de corte ultrassonográficos, das estruturas encefálicas visibilizadas em cada plano de corte obtido por meio de exame ultrassonográfico realizado pela janela occipital nos 30 animais in vivo, e das estruturas visibilizadas nos planos de corte anatômicos correspondentes realizados nos 7 cadáveres. UNESP - Jaboticabal/ 2010

\begin{tabular}{|c|c|c|}
\hline \multirow{2}{*}{$\begin{array}{l}\text { Planos de corte } \\
\text { ultrassonográficos }\end{array}$} & \multicolumn{2}{|c|}{ Estruturas encefálicas visibilizadas } \\
\hline & Corte ultrassonográfico & Corte anatômico \\
\hline Plano longitudinal & $\begin{array}{c}\text { Medula oblonga, cerebelo, vermes } \\
\text { do cerebelo, interface entre a } \\
\text { substância branca e a cinzenta }\end{array}$ & $\begin{array}{l}\text { Medula oblonga, cerebelo, vermes do } \\
\text { cerebelo, interface entre a substância } \\
\text { branca e a cinzenta }\end{array}$ \\
\hline Plano dorsal & $\begin{array}{l}\text { Osso occipital, tentório ósseo do } \\
\text { cerebelo, cerebelo, vermes do } \\
\text { cerebelo }\end{array}$ & $\begin{array}{l}\text { Osso occipital, tentório ósseo do } \\
\text { cerebelo, cerebelo, vermes do } \\
\text { cerebelo }\end{array}$ \\
\hline Plano dorsal oblíquo & $\begin{array}{l}\text { Espaço subaracnóideo, medual, } \\
\text { corno dorsal da medula, corno } \\
\text { ventral da medula, canal central, } \\
\text { corpo do Atlas. }\end{array}$ & $\begin{array}{l}\text { Espaço subaracnóideo, medual, corno } \\
\text { dorsal da medula, corno ventral da } \\
\text { medula, substância intermédia, canal } \\
\text { central, corpo do Atlas. }\end{array}$ \\
\hline
\end{tabular}

Com relação ao exame realizado pela janela occipital, sem a interposição óssea, não foi observada diferença significativa $(p>0,05)$ entre o número de estruturas passíveis de visibilização no exame ultrassonográfico e o número de estruturas presentes nos cortes anatômicos.

\section{DISCUSSÃO}

A interpretação adequada de um exame ultrassonográfico deve fundamentar-se no conhecimento anatômico da região a ser avaliada. Todavia, poucos trabalhos descrevem a anatomia ultrassonográfica dessa região (Hudson 
et al., 1989; Lorigados, 2008). Até o momento não foi possível verificar na literatura compilada a publicação de trabalhos que descrevam a anatomia sonográfica encefálica em planos oblíquos, obtida por meio da região temporal como janela acústica.

As vantagens da USTC observadas na realização deste trabalho comprovam os descritos na literatura (Schellinger et al., 1985; Vachon e Mikity, 1987; Saito, 2001; Berg et al., 2008). Os 30 animais in vivo utilizados não ofereceram nenhuma resistência à contenção para avaliação ultrassonográfica pela janela temporal e à flexão ventral do pescoço para avaliação pela janela occipital, o que permitiu a realização da técnica sem a necessidade de anestesia prévia. Além disso, foi possível constatar a rapidez na realização da técnica, uma vez que o tempo despendido, por um observador previamente treinado, para a avaliação ultrassonográfica completa do encéfalo, foi de 15 minutos para cada animal.

A USTC pela região temporal e occipital foi realizada, neste trabalho, com transdutor convexo multifrequencial, variando em torno de 4 a $7 \mathrm{MHz}$, o que permitiu a obtenção de imagens com boa resolução, detalhamento e definição das estruturas encefálicas, concordando com os relatos da literatura veterinária (Saito et al., 2001; Lorigados, 2008).

Concordando com os achados de Lorigados (2008), neste trabalho também foi observado que, para uma varredura adequada do encéfalo, pela janela temporal, é necessária a realização do exame tanto do lado direito quanto do esquerdo, uma vez que artefatos de reverberação dificultam a avaliação do lado contralateral ao examinado. Esses artefatos se traduziram pela presença de imagens lineares hiperecogênicas presentes no campo proximal da tela, em algumas das imagens obtidas neste trabalho, e que não foram encontradas nos cortes anatômicos correspondentes aos planos de imagens supracitados.

Neste estudo foi possível obter imagens do encéfalo pela janela temporal, em todos os animais da amostra, constatando-se correlação positiva entre as estruturas identificadas em cortes anatômicos com aquelas visibilizadas durante o exame, corroborando os relatos da literatura (Berland et al., 1988; Saito et al., 2001; Lorigados, 2008) e ratificando que o osso temporal, por ser um osso compacto e de espessura fina, pode ser utilizado como janela acústica na realização de USTC em cães adultos de pequeno porte.

Os sulcos encefálicos foram localizados como linhas hiperecogênicas onduladas, cuja ecogenicidade elevada deve-se à interface criada pela parede dos vasos e piamáter. O parênquima encefálico hipoecogênico impediu a diferenciação entre a substância branca e a cinzenta (Hudson et al., 1989; Gallagher et al., 1995).

$\mathrm{Na}$ USTC realizada pela janela temporal por meio de cortes dorsais oblíquos craniais, o número de estruturas observadas nos planos ultrassonográficos foi semelhante $(p>0,05)$ ao observado nos cortes anatômicos nos cadáveres, demonstrando a elevada acurácia dessa técnica. Em contrapartida, nos planos dorsais e dorsais oblíquos caudais, o número de estruturas identificadas nas imagens ultrassonográficas foi significativamente menor $(\mathrm{p}<0,05)$ em relação ao observado nos cortes anatômicos, indicando que, para esses cortes, a perda da definição da imagem, causada pela atenuação do feixe sonoro ao atravessar a interface de tecidos moles com a superfície óssea do crânio, dificulta a diferenciação de algumas estruturas encefálicas. Porém, é de suma importância a inclusão desses cortes na técnica de varredura, uma vez que, por meio deles, em todos os animais avaliados, foi possível observar com detalhes os ventrículos laterais, o septo pelúcido e sulcos encefálicos.

A abordagem ultrassonográfica pelo forame magno permitiu a obtenção de imagens com boa definição e riqueza de detalhes à semelhança ( $p>0,05)$ dos cortes anatômicos, uma vez que não há interposição óssea nesta região anatômica. Por meio desta janela acústica, tal como descrito na literatura (Saito et al., 2001; Lorigados, 2008), foi possível observar apenas a porção caudal do encéfalo (cerebelo, medula oblonga e porção rostral da medula espinhal), sendo necessário, portanto, para uma varredura encefálica completa, o uso da janela occipital em associação com outras janelas acústicas (janela temporal ou fontanelas). 
Ainda com relação à janela occipital, observouse neste trabalho, assim como Saito et al. (2001), a necessidade de flexionar ventralmente o pescoço dos animais para tornar possível o acesso ao forame magno. Essa manobra deve ser realizada com cautela em determinadas raças de cães de pequeno porte por apresentarem anomalias congênitas na região occipitoatlantoaxial. É importante que, mediante essa suspeita clínica, o exame radiográfico da coluna cervical seja realizado antes da USTC.

\section{CONCLUSÃO}

Mediante a técnica utilizada e os resultados obtidos neste estudo, é possível concluir que a USTC é um método diagnóstico viável na avaliação do encéfalo de cães de pequeno porte, com peso corporal até $10 \mathrm{~kg}$, sem a necessidade de anestesiar o paciente. Para a varredura completa do encéfalo, a técnica ultrassonográfica deve consistir de planos dorsal e dorsais oblíquos pelas janelas temporal direita e esquerda e de planos longitudinais, dorsal e dorsal oblíquo pela janela occipital.

\section{AGRADECIMENTOS}

Agradecemos à Prof ${ }^{a}$. Dra. Irvênia Luiza Santis Prada, da Universidade de São Paulo (USP), Prof ${ }^{a}$. Dra. Márcia Rita Fernandez Machado, Narciso Batista Tel, Prof. Dr. Alvimar José da Costa, Prof ${ }^{a}$. Dra. Rosângela Zacarias Machado e Prof. Dr. José Carlos Barbosa, da Universidade Estadual Paulista (UNESP/Jaboticabal), e à M. V. Carla Marconato, pelo auxílio na execução deste trabalho, fornecimento do material necessário e análise dos dados. Este trabalho foi financiado pela Fundação de Amparo à Pesquisa do Estado de São Paulo (FAPESP).

\section{REFERÊNCIAS}

BERLAND, L.L.; BRYAN, C.R.; SEKAR, B.C. Sonographic examination of the adult brain. J. Clin. Ultrasound., v.16, p.337-345, 1988.

BERG, D.; GODAU, J.; WALTER, U. Transcranial sonography in movement disorders. Lancet. Neurol., v.7, p.1044-1055, 2008.
CARVALHO, C.F.; CHAMMAS, M.C.; ANDRADE NETO, J.P. et al. Ultra-Sonografia transcraniana em cães com distúrbios neurológicos de origem central (Transcranial ultrasonography in dogs with central neurologycal disorders). Arq. Bras. Med. Vet. Zootec., v.59, p.1412-1416, 2007.

GALLAGHER, J.G.; PENNINCK, D.; BOUDRIEAU, R.J. et al. Ultrasonography of the brain and vertebral canal in dogs and cats: 15 cases (1988-1993). J. Am. Vet. Med. Assoc., v.207, p.1320-1324, 1995.

HUDSON, J.A.; CARTEE, R.E.; SIMPSON, S.T. et al. Ultrasonographic anatomy of the canine brain. Vet. Radiol., v.30, p.13-21, 1989.

HUDSON, J.A.; FINN-BODNER, S.T.; STEISS, J.E. Neurosonography. Vet. Clin. North. Am. Small. Anim. Pract., v.28, p.943-972, 1998.

LEKSELL, L. Echoencephalography. I. Detection of intracranial complications following head injury. Acta. Chir. Scand., v.110, p.301-315, 1956.

LORIGADOS, C.A.B. Utilização da ultrassonografia modo $B$ e da tomografia computadorizada no estudo do encéfalo de cães adultos. 2008. 90f. Tese (Doutorado em Medicina Veterinária) - Faculdade de Medicina Veterinária, Universidade de São Paulo, São Paulo.

PRYNN, R.B.; REDDING, R.W. Electroencephalogram in occult canine hydrocephalus. J. Am. Vet. Med. Assoc., v.152, p.1651-1657, 1968.

RODRIGUEZ, M.J.; SOLER, M.; LATORRE, L. et al. Ultrasonographic anatomy of the temporomandibular joint in healthy pure-bred Spanish horses. Vet. Radiol. Ultrasound., v.48, p.149-154, 2007.

SAITO, M.; OLBY, J.N.; SPAULDING, K. Identification of arachnoid cysts in the quadrigeminal cistern using ultrasonography. Vet. Radiol. Ultrasound, v.42, p.435-439, 2001.

SCHELLINGER, D.; GRANT, E.G.; RICHARDSON, J.D. Neonatal leukencephalopathy: a common form of cerebral ischemia. Radiographics., v.5, p.221-242, 1985.

VACHON, L.; MIKITY, V. Computed tomography and ultrasound in purulent ventriculitis. J. Ultrasound Med., v.6, p.269-271, 1987. 